\title{
Influence of clove oil and eugenol on muscle contraction of silkworm (Bombyx mori)
}

\author{
Kantaporn Kheawfu', Surachai Pikulkaew ${ }^{2, *}$, Hiroshi Hamamoto ${ }^{3,4}$, Kazuhisa Sekimizu ${ }^{3,4}$, \\ Siriporn Okonogi ${ }^{5,6, *}$
}

${ }^{1}$ Nanoscience and Nanotechnology Program, The Graduate School, Chiang Mai University, Chiang Mai, Thailand;

${ }^{2}$ Department of Food Animal Clinic, Faculty of Veterinary Medicine, Chiang Mai University, Chiang Mai, Thailand;

${ }^{3}$ Laboratory of Microbiology, Graduate School of Pharmaceutical Sciences, The University of Tokyo, Tokyo, Japan;

${ }^{4}$ Teikyo University Institute of Medical Mycology, Tokyo, Japan;

${ }^{5}$ Department of Pharmaceutical Sciences, Faculty of Pharmacy, Chiang Mai University, Chiang Mai, Thailand;

${ }^{6}$ Research Center of Pharmaceutical Nanotechnology, Chiang Mai University, Chiang Mai, Thailand.

\begin{abstract}
Summary Clove oil is used in fish anesthesia and expected to have a mechanism via glutamic receptor. The present study explores the activities of clove oil and its major compound, eugenol, in comparison with L-glutamic acid on glutamic receptor of silkworm muscle and fish anesthesia. It was found that clove oil and eugenol had similar effects to L-glutamic acid on inhibition of silkworm muscle contraction after treated with D-glutamic acid and kainic acid. Anesthetic activity of the test samples was investigated in goldfish. The results demonstrated that L-glutamic acid at 20 and $40 \mathrm{mM}$ could induce the fish to stage 3 of anesthesia that the fish exhibited total loss of equilibrium and muscle tone, whereas clove oil and eugenol at $60 \mathrm{ppm}$ could induce the fish to stage 4 of anesthesia that the reflex activity of the fish was lost. These results suggest that clove oil and eugenol have similar functional activities and mechanism to L-glutamic acid on muscle contraction and fish anesthesia.
\end{abstract}

Keywords: Clove oil, eugenol, glutamic acid, kainic acid, silkworm

\section{Introduction}

Clove oil, an essential oil obtained from buds of clove tree (Syzygium aromaticum), has been traditionally used as topical anesthetic for toothaches, headaches, joint pain. It is also used as an alternative fish anesthetic $(1,2)$, according to its ease of obtaining and inexpensiveness as well as safety for both fish and human (3).

The major component of clove oil is eugenol, which is about $90-97 \%$ of total oil. Both clove oil

Released online in J-STAGE as advance publication April 30, 2017.

*Address correspondence to:

Dr. Siriporn Okonogi, Department of Pharmaceutical Sciences, Faculty of Pharmacy, Chiang Mai University, Chiang Mai, Thailand.

E-mail: okng2000@gmail.com

Dr. Surachai Pikulkaew, Department of Food Animal Clinic, Faculty of Veterinary Medicine, Chiang Mai University, Chiang Mai, Thailand.

E-mail: surapikulkaew@gmail.com and eugenol have been used to anesthetize rainbow trout (Oncorhynchus mykiss) (4), red pacu (Piaractus brachypomus) (5), beluga (Huso huso) (6), nile tilapia (Oreochromis niloticus) (7), and flowerhorn fish (Amphilophus labiatus $\times$ Amphilophus trimaculatus) (8). Furthermore, eugenol can be used in other aquatic animals such as Indian shrimp (Fenneropenaeus indicus) (9), whiteleg shrimp (Litopenaeus vannamei) (10), and the amphibians as African clawed frog (Xenopus laevis) (11), as well as mammals like rat and mouse $(12,13)$.

Even both compounds are widely used for anesthesia of aquatic animals, the anesthetic mechanism pathway of clove oil or eugenol is unclear in fish, but a hypothesis is expectedly via gamma-aminobutyric acid (GABA) and glutamate receptors (14). Invertebrate animal models are preferable choice for drug-screening and used instead of vertebrates. They offer a great potential for cost-effective due to culture conditions fulfill the requirements for large-scale screens, less ethical concern, and fundamental biological mechanisms of invertebrates correlate with mammals (15). In case of signal transmission, the receptor of invertebrate 
animals such as cricket (Gryllus domestica) and fruit fly (Drosophila melanogaster) on presynaptic nerve terminals or muscle membranes have a similar pharmacological profile as 5-HT like receptor subtype $(16,17)$. Furthermore, silkworm (Bombyx mori) was discovered that the neurotransmitters of glutamic acid functions acted on their neuromuscular junction. D-glutamic acid and kainic acid as glutamate receptor agonists could induce muscle contraction in silkworm after injected into hemolymph, meanwhile, L-glutamic acid presented as antagonist to the activities of D-glutamic acid and kainic acid. Hence, after injection these glutamate receptor agonists, muscle contraction would be inhibited by injection of L-glutamic acid (18).

It was suspected whether the mechanism of action of clove oil as anesthetic agent was involved in glutamic acid functions. We hypothesized that clove oil and eugenol might have anesthetic activity as glutamate receptor antagonist, and should inhibit muscle contraction induced by both D-glutamic acid and kainic acid.

\section{Materials and Methods}

\subsection{Materials}

Clove oil was purchased from Thai-China Flavours \& Fragrances Industry (Nonthaburi, Thailand). Eugenol was from Sigma-Aldrich (Steinheim, Germany). L-glutamic acid, D-glutamic acid, sodium hydroxide, and ethanol were from Wako Pure Chemical Industries (Osaka, Japan). Kainic acid was from Abcam (Cambridge, UK). Saline was from Otsuka Pharmaceutical (Tokyo, Japan).

\subsection{Silkworm larvae muscle contraction assay}

The assay was performed according to the method previously described by Sekimizu et al. (18). The $5^{\text {th }}$ instar larval stage silkworms, body weight of 4-6 $\mathrm{g}$, were prepared and their heads were cut off with scissors, then peritrophic membranes and silk glands were removed. Each specimen was tied with strings and attached with load of 29-30 g. The test samples of this study, included 20-80 $\mathrm{mM} \mathrm{L-glutamic} \mathrm{acid,} \mathrm{0.9-}$ $90 \%$ ethanol (as vehicle), clove oil containing 6-600 $\mathrm{mM}$ eugenol (namely 6-600 $\mathrm{mM}$ clove oil), and 6-600 $\mathrm{mM}$ pure eugenol, were dispersed in saline and filled in a 1 -mL syringe attached to a 27 -gauge needle (Terumo, Tokyo, Japan). Then, the test samples $(50 \mu \mathrm{L})$ were injected into the silkworm specimens, followed by an injection of $40 \mathrm{mM}$ D-glutamic acid or $0.2 \mathrm{mM}$ kainic acid $(50 \mu \mathrm{L})$. The intensity of muscle contraction was expressed as the contraction value calculated by the following equation; $(x-y) / x$. Where $x$ and $y$ are the maximum length $(\mathrm{mm})$ of each individual specimen before and after injection, respectively.

\subsection{Fish anesthetic experiment}

Twenty-four goldfish (Carassius auratus) with an average length of $3.97 \pm 0.33 \mathrm{~cm}$ were stocked separately in 20-L tank and supplied with running and aerated water for 1 week under control conditions. The fish were not fed for $24 \mathrm{~h}$ prior to testing.

For the determination of the anesthetic activity, 8 fish were netted and transferred individually to 8 plastic experimental tanks filled with $2 \mathrm{~L}$ of water from a similar source. Clove oil and eugenol were diluted with ethanol to obtain the desired concentrations. The fish were placed into each tank containing $60 \mathrm{ppm}$ clove oil, $60 \mathrm{ppm}$ (or $0.36 \mathrm{mM}$ ) eugenol, $540 \mathrm{ppm}$ ethanol (as a vehicle control), and $0.4,4,20$, and $40 \mathrm{mM}$ L-glutamic acid. The tank without any test sample was used as a control tank. Induction time, recovery time, and mortality were estimated, as described by Iversen et al. and Iwama et al. $(19,20)$. The induction time is the period from the moment a fish is exposed to the samples until it exhibits anesthesia which can be divided based on fish behavior into 4 stages as followings; no reaction to external stimuli (stage 1), partial loss equilibrium (stage 2), total loss equilibrium and muscle tone (stage 3), and loss of reflex activity (stage 4). After 10 -sec stay in stage 4, the fish were transferred into the identical tank without any anesthetic agent and were observed to determine recovery time or mortality. The recovery time is the time that an anesthetized fish takes in order to regain full equilibrium from the moment it is placed in a recovery tank. The recovery is divided into 3 stages as followings; fish begin to have opercular movement (stage 1), recovery of equilibrium and body movement (stage 2), and full recovery similar to preanesthesia (stage 3). The recovery and induction times were recorded for each fish individually. After recovery, the fish were transferred into the maintenance tanks (20 L) and the adverse effects were observed for one week. Each sample was tested in triplicate.

\subsection{Statistical analysis}

Statistical evaluation of anesthetic induction and recovery times in fish experiment was performed by oneway ANOVA (Bonferroni test). Data were presented as mean $\pm \mathrm{SD}$. The value of $p<0.05$ was considered to indicate significant differences.

\section{Results}

\subsection{Effect of eugenol on D-glutamic acid-induced muscle contraction in silkworm}

We re-examined the inhibitory effect of L-glutamic acid on D-glutamic acid followed the established silkworm muscle contraction experiment. D-glutamic acid is an amino acid existed in silkworm hemolymph and 


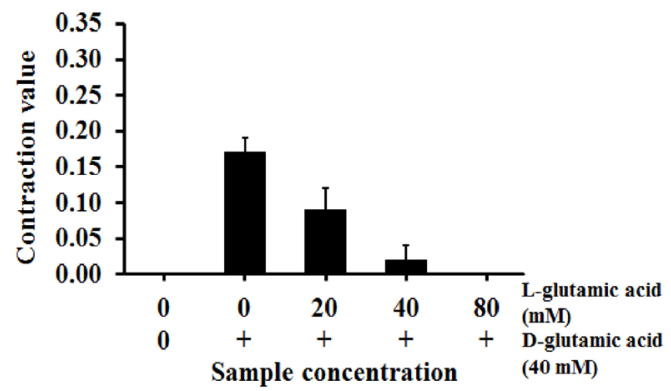

Figure 1. The effect of L-glutamic acid on D-glutamic acid induced silkworm muscle contraction $(n \geq 2)$.

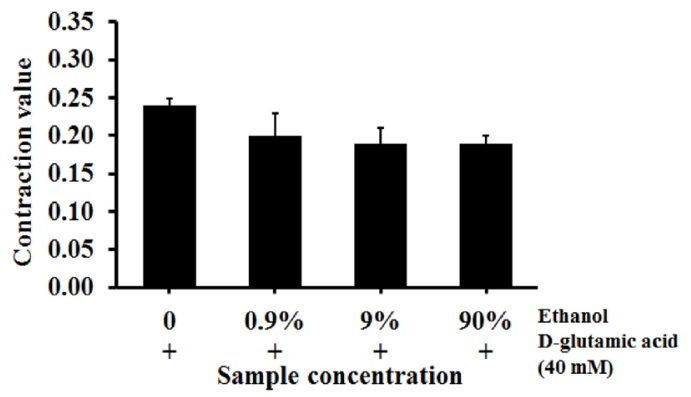

Figure 2. The effect of ethanol on D-glutamic acid induced muscle contraction in silkworm $(n \geq 2)$.

thought to regulate the muscle contraction in silkworm. A single injection of D-glutamic acid in decapitated silkworm caused muscle contraction with a contraction value of 0.25 within $1 \mathrm{~min}$. After $3 \mathrm{~min}$ of this contraction, slightly muscle relaxation was observe and fully relaxation was found in $30 \mathrm{~min}$. Pre-injection with L-glutamic acid showed inhibition on the D-glutamic acid induced muscle contraction as shown in Figure 1, as previously reported (18).

We next examined the action of ethanol, which was used to dissolve clove oil and eugenol to the desired concentrations before dispersing in water for fish anesthesia. The ethanol did not show any effect on D-glutamic acid induced muscle contraction, as presented in Figure 2. Pretreatment of the muscle specimen with clove oil and eugenol showed inhibition of the muscle contraction induced by D-glutamic acid, similar to the pretreatment with L-glutamic acid. However, there was some difference in this inhibitory effect. Silkworm larvae injected with clove oil and eugenol followed by D-glutamic acid firstly showed a short spasmodic contraction for about $2 \mathrm{sec}$ with a contraction value of 0.18 and then fully muscle relaxation was observed. In contrast, L-glutamic acid suddenly showed the inhibitory effect on D-glutamic acid induced muscle contraction, so that the entire muscle relaxation in silkworm larvae was observed. However, after $10 \mathrm{~min}$, the inhibitory activity of L-glutamic acid was disappeared and some slight muscle contraction was observed. Figure 3 showed that the inhibitory activity of clove oil and eugenol on D-glutamic acid induced muscle contraction was dose
(A)

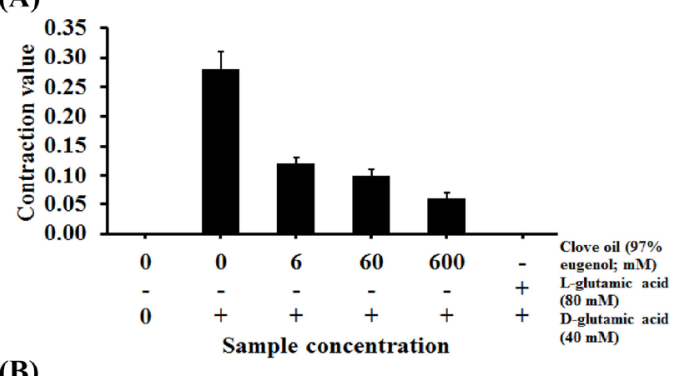

(B)

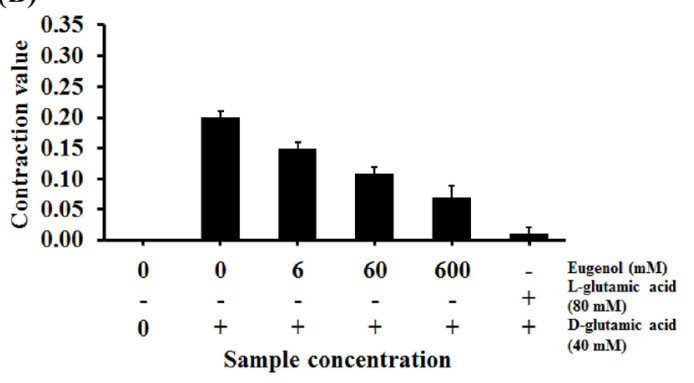

Figure 3. Contraction value of silkworm injected with clove oil (A) and eugenol (B) followed by D-glutamic acid $(n=2)$.

dependent. From these results, percent inhibition of 6-600 $\mathrm{mM}$ clove oil and eugenol could be calculated and it was found to be $57-79 \%$ and $25-65 \%$, respectively. It is concluded that clove oil and eugenol possessed inhibitory effect on D-glutamic acid-induced muscle contraction.

\subsection{Effect of eugenol on kainic acid-induced muscle contraction in silkworm}

Kainic acid, a glutamate receptor agonist, can also induce muscle contraction of decapitated silkworm. Its activity is stronger than D-glutamic acid. A single injection of kainic acid in decapitated silkworm caused muscle contraction with a contraction value of 0.27 within $1 \mathrm{~min}$. After 5-10 min of this contraction, slightly muscle relaxation was observed and fully relaxation was found in $30 \mathrm{~min}$. Ethanol displayed no effect on the contraction by kainic acid. Prior injection of clove oil and eugenol into silkworm larvae inhibited muscle contraction induced by kainic acid in dose-dependent manner, similar to that induced by D-glutamic acid. However, the inhibitory action resulted from clove oil and eugenol on muscle contraction induced by kainic acid was lower than that induced by D-glutamic acid. This might be due to the stronger activity of kainic acid than D-glutamic acid. Silkworm larvae injected with clove oil and eugenol followed by kainic acid firstly showed a slight spasmodic contraction for about 5-10 sec with a contraction value of 0.15 and then fully muscle relaxation was gradually observed. The results in Figure 4 confirmed that clove oil and eugenol inhibited muscle contraction induced by kainic acid. The inhibition was found to be $37-70 \%$ and $24-$ $59 \%$, respectively, slightly lower than that induced by D-glutamic acid. From this experiment, it could 
be concluded that clove oil and eugenol possessed the inhibitory effect on kainic acid-induced muscle contraction.

\subsection{Fish anesthetic effect of L-glutamic acid}

Clove oil and eugenol at $60 \mathrm{ppm}$ induced goldfish anesthesia to stage 4 of anesthetic induction within 6 $\mathrm{min}$, and the fish recovered within $10 \mathrm{~min}$. L-glutamic acid at 20 and $40 \mathrm{mM}$ also induced goldfish anesthesia but to stage 3 within 35 and $25 \mathrm{~min}$, respectively, and the

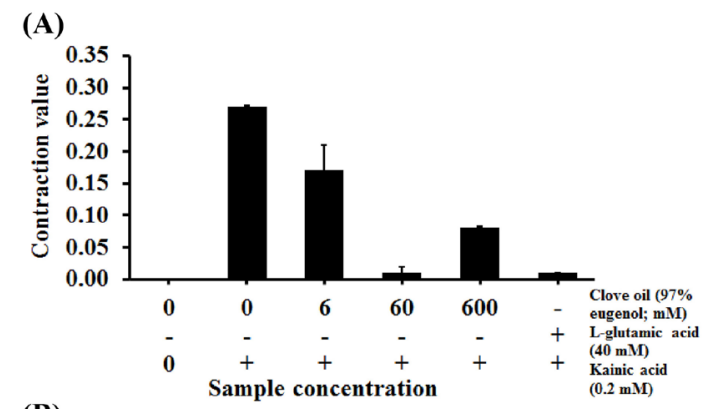

(B)

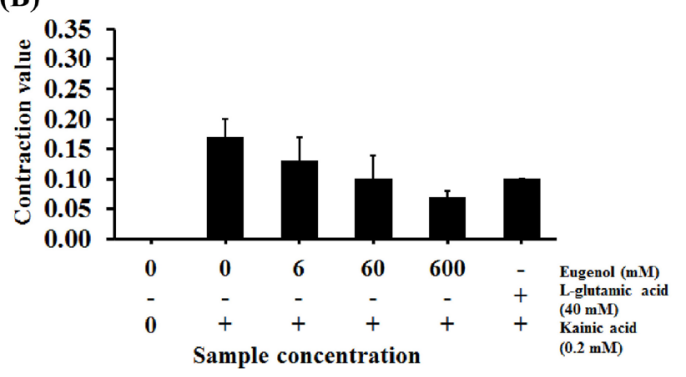

Figure 4. The effect of clove oil (A) and eugenol (B) on the kainic acid-induced silkworm muscle contraction $(n=2)$.
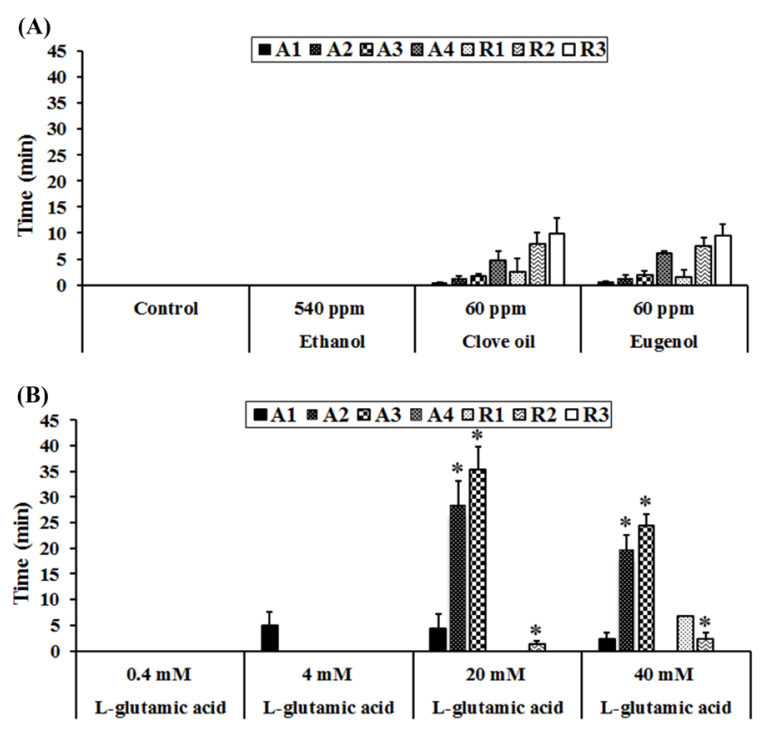

Figure 5. Anesthetic induction and recovery time of control, ethanol, clove oil and eugenol (A), L-glutamic acid (B) (n $=3)$. $\mathrm{A} 1$ to $\mathrm{A} 4$ represented the anesthetic induction stage 1 to stage 4 , respectively and R1 to R3 represented the recovery at stage 1 to stage 3 , respectively. ${ }^{*} p<0.05$ compared to clove oil and eugenol groups. fish could not recover but died. The goldfish received 20 and $40 \mathrm{mM}$ L-glutamic acid significantly showed longer induction and shorter recovery times than those received clove oil and eugenol. Moreover, the fish which received L-glutamic acid at these concentrations were anesthetized to only stage 3 , whereas those received clove oil and eugenol could be anesthetized to stage 4 of anesthesia. The recovery behavior was also different. The anesthetized fish which received L-glutamic acid could recover to only stage 2 and then died after that, whereas those received clove oil and eugenol could fully recover to stage 3. Furthermore, it was found that L-glutamic acid at a concentration of $4 \mathrm{mM}$ could induce anesthetic effect in goldfish within 5 min but only to stage 1, after that the fish fully recovered, as exhibited in Figure 5. The obtained results indicated that L-glutamic acid has slight anesthetic effect on fish.

\section{Discussion}

This study demonstrates that clove oil and its main component, eugenol, can inhibit muscle contraction induced by D-glutamic acid and kainic acid in silkworm larvae, similar to the inhibitory effect of L-glutamic acid. Eugenol has been reported to inhibit the nociceptive response caused by glutamate, $\alpha$-amino-3-hydroxy-5methyl-4-isoxazolepropionic acid, and kainite in mice (21). In addition, eugenol showed the inhibitory effect against acetylcholine esterase, an enzyme for hydrolysis the acetylcholine, which can induce paralysis in rice weevil (Sitophilus oryzae) (22).

The chemical structure of eugenol and L-glutamic acid are completely different which the eugenol is a phenylpropene, whereas L-glutamic acid is an amino acid containing amine and carboxylic acid functional groups, as seen in Figure 6. This point leads to some different results between these two compounds that the silkworm muscles after L-glutamic acid injection presented the delayed muscle contraction after relaxation by inhibiting D-glutamic acid and kainic acid action. It is possible that silkworm bodies have enzyme to degrade glutamic acid, which preferably degrades L-glutamic acid to D-glutamic

(A)<smiles>C=CCc1ccc(O)c(OC)c1</smiles>

(B)<smiles>N[C@@H](CCC(=O)O)C(=O)O</smiles>

L-glutamic acid

Figure 6. The chemical structure of eugenol (A) and L-glutamic acid (B). 
acid. Therefore, L-glutamic acid is eliminated faster than D-glutamic acid. Then, the remained D-glutamic acid or kainic acid could show their action of muscle contraction again after L-glutamic acid elimination.

Most of insects have an open circulatory system. The body cavity is filled with hemolymph that bathes the internal organs (23). Our study demonstrates that injection of clove oil and eugenol into silkworm bodies can induce muscle relaxation, and has inhibitory effect on muscle contraction activity of kainic acid and D-glutamic acid which are agonists of glutamate receptor expressed on larval body wall muscles $(18,24)$. The results indicate that clove oil and eugenol have some effects on silkworm glutamate receptor, supporting the effect of these compounds tested in vitro and in vivo in mice $(21,25)$.

In contrast to silkworm, fish can receive the test samples which were dispersed in the water tank for fish via the expected organ like gill. The gill is an important organ for respiratory and circulating system for reoxygenated of blood. After that the blood with active substances flows to the other organs including brain (26), and to the expected GABA subtype A receptors as predominate receptor responds for anesthetic mechanism in fish (27).

The present study firstly demonstrated that L-glutamic acid, a biological agonist excitatory neurotransmitter in the nervous system, had anesthetic effect in fish. It is possible that glutamate receptor also responded on this effect, contributed to mechanism of eugenol. In case of fish, 0.8-1 mM L-glutamic acid induced transient excitation followed by suppression on the isolated Plotosus electroreceptor, contrast with lower dose (0.2$0.3 \mathrm{mM}$ ) induced a sustained excitation (28). Meanwhile, D-glutamic acid induced fictive swimming in the isolated Lamprey spinal cords that closely matches the in vivo swimming pattern, analogous to isolated mammalian spinal cords (29).

However, the goldfish which received L-glutamic acid could not recover. It has been hypothesized that glutamic acid is advantaged substance in hyperammonemic conditions, ammonia was detoxified by glutamic acid and then generated glutamine in astrocyte. However, the excess of glutamine could exert osmotic effects and contribute to brain swelling (30). The prior study showed that glutamine can directly exert toxic effects on cultured astrocytes by increasing reactive oxygen species production and by inducing the mitochondrial permeability transition (31).

This is the first paper to use silkworm larvae for testing fish anesthetic agents and comparing the action of clove oil, eugenol, and L-glutamic acid in both animals. The further investigation is required to challenge with other fish anesthetic agents. It might be possible to use silkworm as model animals for screening new anesthetic agents for fish, since the use of silkworm is low cost, easy breeding, simple handle, and reproducible obtained results (32).
In conclusion, these results suggest that clove oil and eugenol have inhibitory effect on the D-glutamic acid and kainic acid-induced silkworm muscle contraction similar to L-glutamic acid effect. Moreover, L-glutamic acid induced dose dependent anesthetic effects in goldfish similar to clove oil and eugenol.

\section{Acknowledgements}

This work was supported by the Thailand Research Fund (TRF) through the Royal Golden Jubilee PhD Program (RGJ) Grant No 5.NS.CM/56/A.1. We also thank Research Center of Pharmaceutical Nanotechnology, Chiang Mai University, Thailand and Teikyo University, Japan for their support.

\section{References}

1. Bunyapraphatsara N. Clove oil. In: Thai Medicinal Plants (Bunyapraphatsara N, Chokchaijarenporn O., eds.). Prachachon, Bangkok, Thailand, 1996; pp. 211224.

2. Woody CA, Nelson J, Ramstad K. Clove oil as an anaesthetic for adult sockeye salmon: Field trials. J Fish Biol. 2002; 60:340-347.

3. Pirhonen J, Schreck CB. Effects of anaesthesia with MS-222, clove oil and $\mathrm{CO} 2$ on feed intake and plasma cortisol in steelhead trout (Oncorhynchus mykiss). Aquaculture. 2003; 220:507-514.

4. Prince A, Powell C. Clove oil as an anesthetic for invasive field procedures on adult rainbow trout. N Am J Fish Manag. 2000; 20:1029-1032.

5. Sladky KK, Swanson CR, Stoskopf MK, Loomis MR, Lewbart GA. Comparative efficacy of tricaine methanesulfonate and clove oil for use as anesthetics in red pacu (Piaractus brachypomus). Am J Vet Res. 2001; 62:337-342.

6. Hoseini SM, Ghelichpour M. Efficacy of clove solution on blood sampling and hematological study in beluga, Huso Huso (L.). Fish Physiol Biochem. 2012; 38:493498.

7. Ribeiro PAP, Miranda-Filho KC, de Melo DC, Luz RK. Efficiency of eugenol as anesthetic for the early life stages of nile tilapia (Oreochromis niloticus). An Acad Bras Ciênc. 2015; 87:529-535.

8. Tarkhani R, Imani A, Jamali H, Moghanlou, KS. Anaesthetic efficacy of eugenol on Flowerhorn (Amphilophus labiatus $\times$ Amphilophus trimaculatus). Aquacult Res. 2016; 47:1-9.

9. Akbari S, Khoshnod MJ, Rajaian H, Afsharnasab M. The use of eugenol as an anesthetic in transportation of white Indian shrimp (Fenneropenaeus indicus) post larvae. Turk J Fish Aquat Sci. 2010; 10:423-429.

10. Parodi TV, Cunha MA, Heldwein CG, de Souza DM, Martins ÁC, Garcia LO, Wasielesky WJ, Monserrat JM, Schmidt D, Caron BO, Heinzmann B, Baldisserotto B. The anesthetic efficacy of eugenol and the essential oils of Lippia alba and Aloysia triphylla in post-larvae and sub-adults of Litopenaeus vannamei (Crustacea, Penaeidae). Comp Biochem Physiol C Toxicol Pharmacol. 2012; 155:462-468.

11. Goulet F, Vachon P, Hélie P. Evaluation of the toxicity 
of eugenol at anesthetic doses in African clawed frogs (Xenopus laevis). Toxicol Pathol. 2011; 39:471-477.

12. Sell A, Carlini E. Anesthetic action of methyleugenol and other eugenol derivatives. Pharmacology. 1976; 14:367377.

13. Guenette SA, Beaudry F, Marier JF, Vachon P. Pharmacokinetics and anesthetic activity of eugenol in male Sprague-Dawley rats. J Vet Pharmacol Ther. 2006; 29:265-270.

14. Meyer RE, Fish RE. Pharmacology of injectable anesthetics, sedatives, and tranquilizers. In: Anesthesia and Analgesia in Laboratory Animals (Fish RE, Brown MJ, Danneman PJ, Karas AZ, eds.). Elsevier, Massachusetts, USA, 2008; pp. 59-60.

15. Segalat L. Invertebrate animal models of diseases as screening tools in drug discovery. ACS Chem Biol. 2007; 2:231-236

16. Baines RA, Downer RG. Pharmacological characterization of a 5-hydroxytryptamine-sensitive receptor/adenylate cyclase complex in the mandibular closer muscles of the cricket, Gryllus domestica. Arch Insect Biochem Physiol. 1991; 16:153-163.

17. Wu WH, Cooper RL. Serotonin and synaptic transmission at invertebrate neuromuscular junctions. Exp Neurobiol. 2012; 21:101-112.

18. Sekimizu K, Larranaga J, Hamamoto H, Sekine M, Furuchi T, Katane M, Homma H, Matsuki N. D-glutamic acid-induced muscle contraction in the silkworm, Bombyx mori. J Biochem. 2005; 203:199-203.

19. Iversen M, Finstad B, McKinley RS, Eliassen R. The efficacy of metomidate, clove oil, Aqui-S ${ }^{\mathrm{TM}}$ and Benzoak $^{\circledR}$ as anaesthetics in Atlantic salmon (Salmo salar L.) smolts, and their potential stress-reducing capacity. Aquaculture. 2003; 221:549-566.

20. Iwama GK, McGeer JC, Pawluk MP. The effects of five fish anaesthetics on acid-base balance, hematocrit, cortisol, and adrenaline in rainbow trout. Can J Zool. 1989; 67:2065-2073.

21. Dal BW, Luiz AP, Martins DF, Mazzardo-Martins L, Santos ARS. Eugenol reduces acute pain in mice by modulating the glutamatergic and tumor necrosis factor alpha (TNF- $\alpha$ ) pathways. Fundam Clin Pharmacol. 2013; 27:517-525.
22. Lee S, Lee B, Choi W, Park B, Kim J, Campbell BC. Fumigant toxicity of volatile natural products from Korean spices and medicinal plants towards the rice weevil, Sitophilus oryzae (L.). Pest Manag Sci. 2001; 57:548-553.

23. Miller TA, Pass G. Circulatory system. In: Encyclopedia of Insects (Resh VH, Cardé RT, eds.). Academic Press, Massachusetts, USA, 2009; pp. 169-173.

24. Ishii K, Hamamoto H, Sasaki T, Ikegaya Y, Yamatsugu K, Kanai M, Shibasaki M, Sekimizu K. Pharmacologic action of oseltamivir on the nervous system. Drug Discov Ther. 2008; 2:24-34.

25. Won MH, Lee JC, Kim YH, Song DK, Suh HW, Oh YS, Kim JH, Shin TK, Lee YJ, Wie MB. Postischemic hypothermia induced by eugenol protects hippocampal neurons from global ischemia in gerbils. Neurosci Lett. 1998; 254:101-104.

26. Sneddon LU. Clinical anesthesia and analgesia in fish. J Exot Pet Med. 2012; 21:59-70.

27. Heldwein CG, Silva LL, Gai EZ, Roman C, Parodi TV, Bürger ME, Baldisserotto B, Flores ÉM, Heinzmann BM. S-(+)-Linalool from Lippia alba: Sedative and anesthetic for silver catfish (Rhamdia quelen). Vet Anaesth Analg. 2014; 41:621-629.

28. Okano K, Obara S. Effects of bath-applied L-glutamate and related chemicals on the afferent synapse of the Plotosus electroreceptor. Brain Res. 1988; 457:79-88.

29. Jinks SL, Andrada J. Validation and insights of anesthetic action in an early vertebrate network: The isolated lamprey spinal cord. Anesth Analg. 2011; 113:10331042.

30. Ip YK, Chew SF. Ammonia production, excretion, toxicity, and defense in fish: A review. Front Physiol. 2010; 1:1-20.

31. Jayakumar AR, Rama Rao KV, Schousboe A, Norenberg MD. Glutamine-induced free radical production in cultured astrocytes. Glia. 2004; 46:296-301.

32. Sekimizu N, Paudel A, Hamamoto H. Animal welfare and use of silkworm as a model animal. Drug Discov Ther. 2012; 6:226-229.

(Received February 18, 2017; Revised April 16, 2017; Accepted April 20, 2017) 